\title{
High Power 650 MHz Magnetron RF Power Source
}

Thomas Kroc, Ram Dhuley, Jayakar Thangaraj - Fermilab NSARD2021

21 April, 2021 


\section{Technical approach: SRF tech and Fermilab}

- Fermilab is a world leader in SRF technology for discovery science as demonstrated by delivering SRF cryomodules for LCLS-II and building PIP-II.

- SRF is a mature technology, however, to be useful in non-scientific applications, parameters such as compactness and simplicity need to be considered.

- The elimination of liquid cryogens makes SRF technology accessible for industrial applications. This can be accomplished through Conduction Cooling which has been demonstrated by Fermilab.

- The first year of this effort (FY20) investigated RF sources for SRF applications.

- Now we are integrating conduction cooling and related technologies for the first time into a prototype accelerator as part of alternative technology for Co-60 replacement for medical device sterilization. 


\section{Capability Improvement}

Efficient, high-power X-ray source for medical device sterilization

- 1 Mci of Co-60 produces $\sim 15 \mathrm{~kW}$ of power

- X-rays are essentially a direct replacement for Co-60 irradiation

- Due to Bremsstrahlung inefficiency, need $\sim 120 \mathrm{~kW}$ of electron beam power to equal 1 Mci of Co-60

- Medical device production is growing by $\sim 7 \%$ per year. To provide comparable treatment capacity, additional accelerator designs are needed. Enter SRF.

- Achieving this as compactly, simply, and efficiently as possible will improve the attractiveness of alternative technologies to help move the industry away from Co-60.

- Validation of this design is the final step before a first article of $7.5 \mathrm{MeV}$, $150-200 \mathrm{~kW}$. 


\section{Project Overview, Goals, Deliverables}

Goal: Fabricate and assemble a prototype 1.6 MeV compact SRF system to validate the integration of:

- Integrated electron gun

- Conduction cooling \& Cryocoolers

- $\mathrm{Nb}_{3}$ Sn coating

- Low-heat loss RF coupler

- Deliverables

- FY21 - RF Coupler

- FY22 - Cavity \& Cryostat

- FY23 - coated \& tested cavity, commissioned system 


\section{Progress to Date}

We are in the process of finalizing design work of major components

1. Gun design

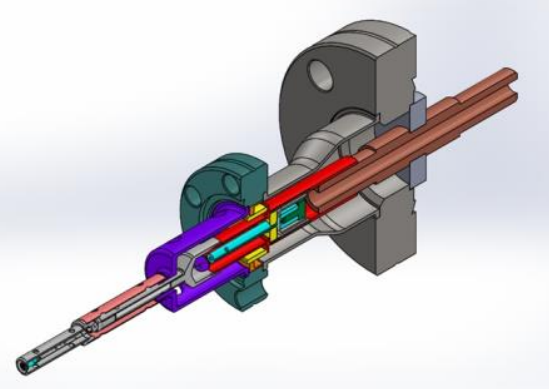

- Lengthening to improve thermal distribution

- Impacts Cryostat design
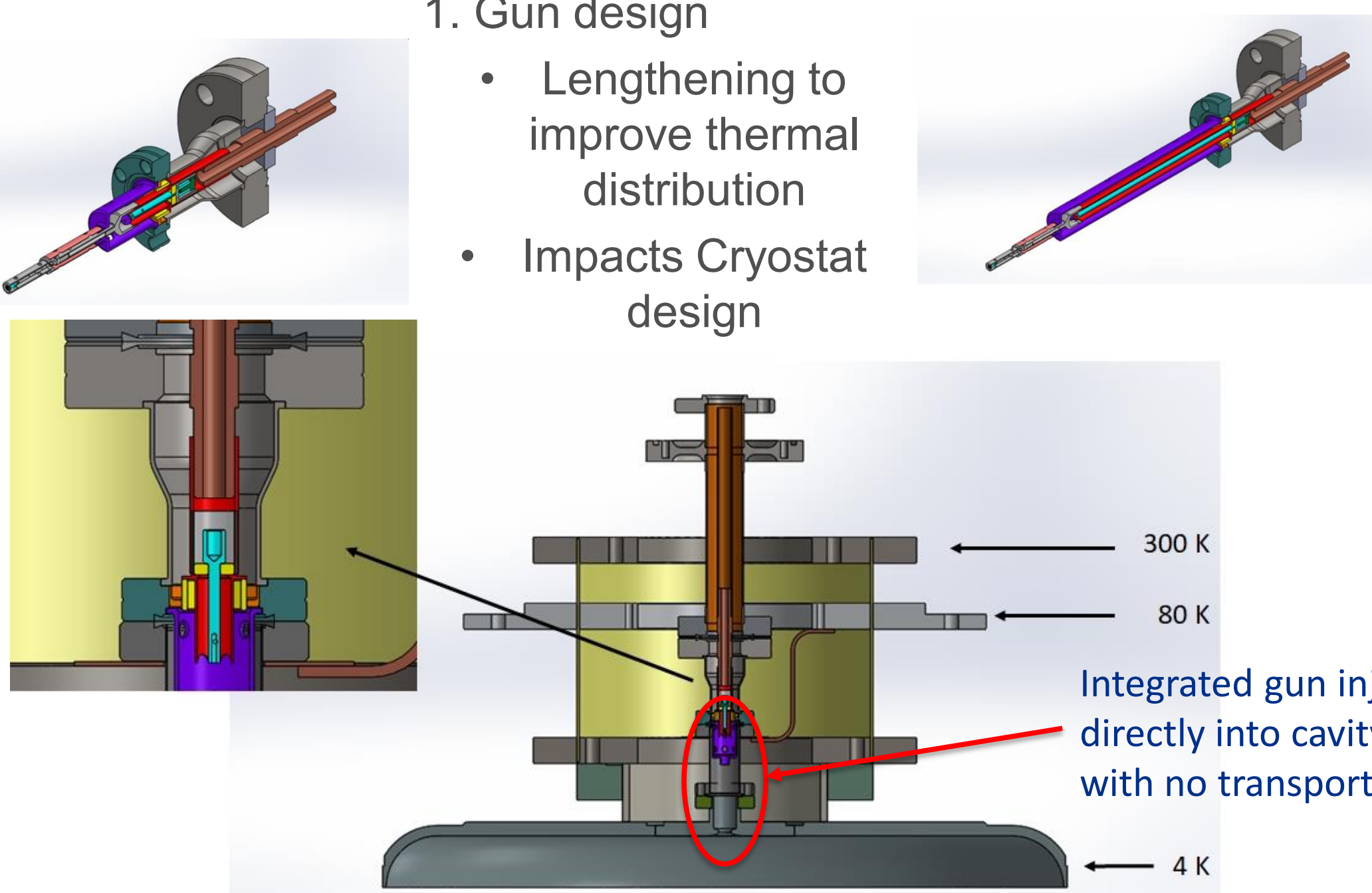

Integrated gun injects directly into cavity with no transport line 


\section{Progress to Date}

Finalizing design work of major components

2. Cavity Design

- Completed

- RF and beam transport simulations

- Structural design

- Niobium material procurement

- In progress

- Production drawings

- Fabrication bid package

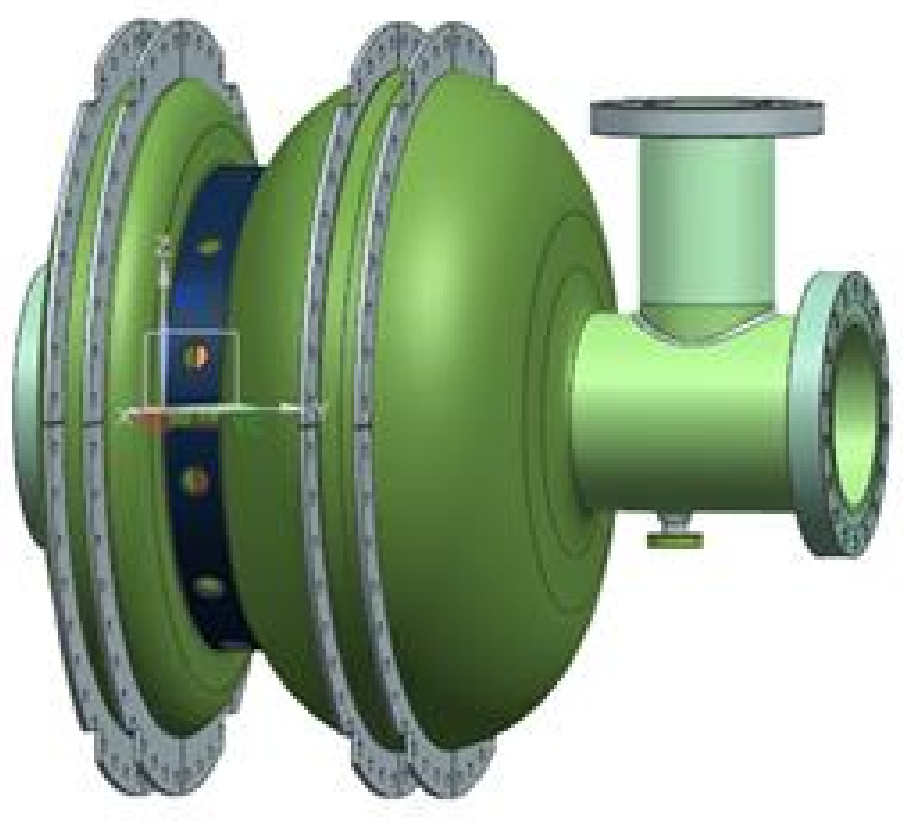




\section{Progress to Date}

Finalizing design work of major components

3. Cryostat Design

- Completed 3D modeling of all components

- Vacuum jacket

- Thermal and magnetic shields

- Cavity support, coupler interface

- In progress

- FEA analyses of the above components

- Next

- Fabrication drawing and bid package

- Cryomech PT425 now available (2.5 W@ @ 4.2 K)

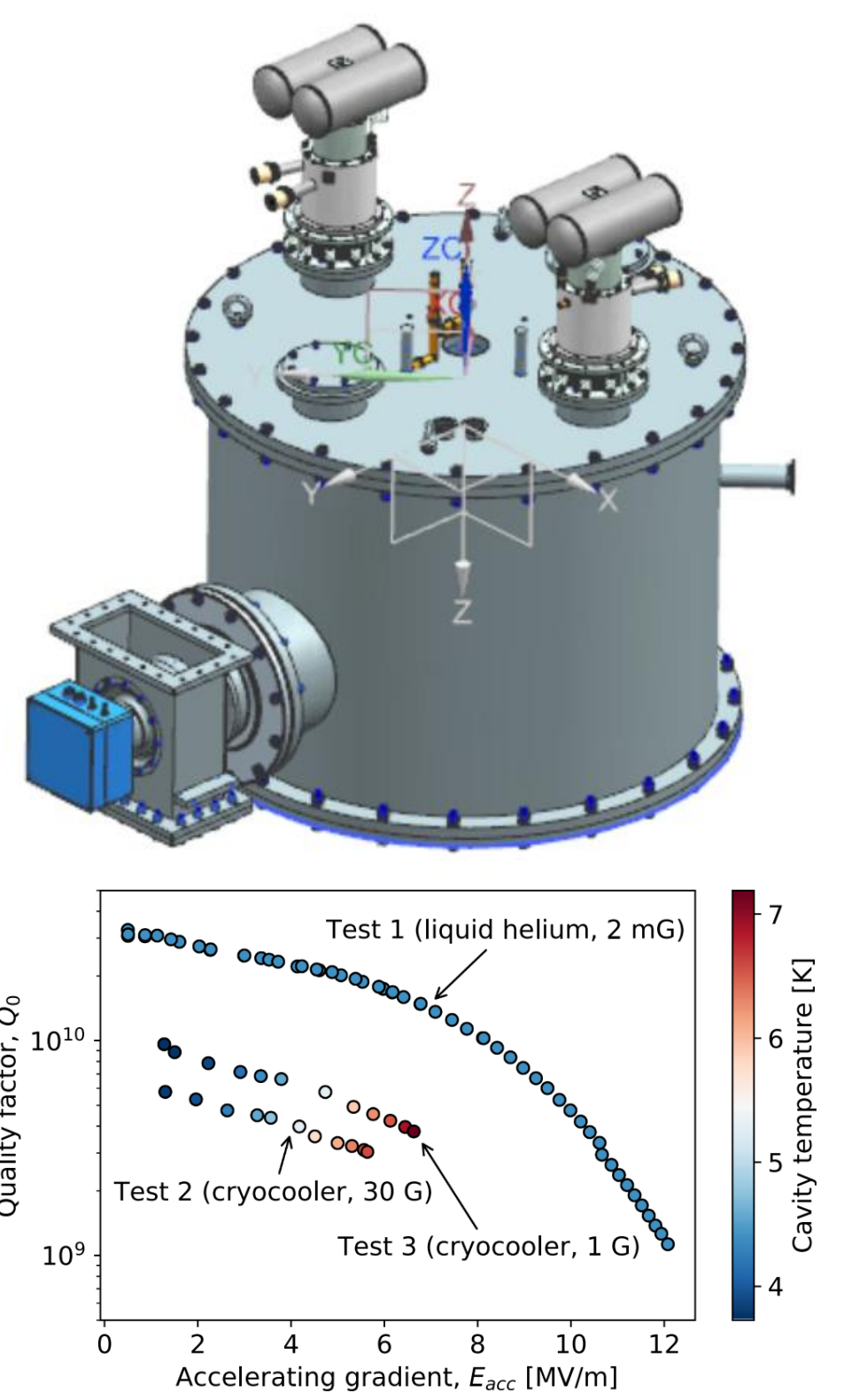




\section{Nonproliferation}

Presently, X-ray sterilization represents about $1 \%$ of the medical device sterilization market whereas Co-60 services $40-50 \%$.

- However, 7 new X-ray facilities have been announced and will become operational in the next two years.

- Capacity issues in the medical device sterilization market are driving new interest in alternative technologies.

- A forecast presented at the 2019 Midwest Medical Device Sterilization Workshop projected the need for $200-$ 400 new accelerator systems in the next 10 years. * 


\section{Technical Challenges}

- Coating of single-ended cavity

- Double ended cavities have been coated very reliably by Fermilab with excellent performance.

- The coating process continues to be aggressively developed

- Our coating needs are still 18 months away.

- Determination of dynamic losses (under RF and with beam)

- This will be a fundamental validation of the heat budget that enables the compact SRF concept.

- Economical RF power

- Not part of this effort, but cost of RF power will become the major single expense of an accelerator system. 


\section{Future Work}

FY21:

- The majority of work in this year is procurement of long-lead items.

- Other items are preparation of the test cave and acquiring diagnostics

FY22

- Testing of RF supplies \& bare cavity. Begin $\mathrm{Nb}_{3} \mathrm{Sn}$ coating. FY 23

- Final integration and commissioning 